\title{
Representaciones sociales del cuidado de la salud sexual y reproductiva en estudiantes de enfermería
}

Social representations of sexual and reproductive healthcare in nursing students

Representações sociais de cuidados de saúde sexual e reprodutiva em estudantes de enfermagem

Jesús Roberto Garay Núñez

Escuela Superior de Enfermería Culiacán, Universidad Autónoma de Sinaloa, México jrgarayn@hotmail.com

María Araceli Félix Amezquita

Escuela Superior de Enfermería Culiacán, Universidad Autónoma de Sinaloa, México aracelifelix56@hotmail.com

María Isabel Santos Quintero

Escuela Superior de Enfermería Culiacán, Universidad Autónoma de Sinaloa, México misq@hotmail.com

\section{Resumen}

La presente investigación tuvo como objetivos de estudio: describir el núcleo central y núcleo periférico de cada una de las representaciones sociales de la salud sexual y reproductiva en estudiantes de la Escuela Superior de Enfermería Culiacán, México, y analizar las diferencias entre hombres y mujeres. El abordaje teórico se basó en los conceptos sobre la teoría de las representaciones sociales de Moscovici (2000), y los conceptos principales sobre cuidado Waldow (2004), salud sexual y reproductiva (SSA/México, 2006) guiarán el abordaje teórico de la presente investigación. Con abordaje metodológico cuantitativo se aplicaron cuestionarios semi estructurados para el enfoque estructural. Para el análisis de la información se usó el análisis del núcleo central de Abric 
(1994). Los resultados desvelaron tres categorías: I) Cuidado de la salud sexual y reproductiva en el embarazo no deseado femenino y masculino. II) Cuidado de la salud sexual y reproductiva en los métodos anticonceptivos femenino y masculino. Y III) Cuidado de la salud sexual y reproductiva en las enfermedades de trasmisión sexual femenino y masculino. Con cuatro sub-categorías: 1) Relaciones con varias parejas, 2) Educación sexual ausente, 3) Pensamientos de a mí no me va a pasar, 4) Alcohol y drogas.

Palabras clave: representaciones sociales, cuidado, salud sexual y reproductiva.

\section{Abstract}

The present research had as objectives of study: describe the central and peripheral nucleus of each of the social representations of sexual and reproductive healthcare in students of the Higher Nursing School of Culiacán, México, and analyze the differences between men and women. The theoretical approach was based on the concepts on the theory of social representations of Moscovici (2000), and the main concepts of healthcare Waldow (2004), sexual and reproductive healthcare (SSA/México, 2006); these will guide the theoretical approach of this research. With quantitative methodological approach applied questionnaires semi-structured for the structural approach. The analysis of central core of Abric (1994) was used for the analysis of the information. The results revealed three categories: I) Sexual and reproductive healthcare in male and female unwanted pregnancy. II) Sexual and reproductive healthcare in male and female methods of contraception. III) Sexual and reproductive healthcare in male and female sexually transmitted diseases. With four sub-categories: 1) Multiple-partner relationships, 2) Absent sex education, 3) Thoughts of "It won't happen to me", 4) Alcohol and drugs.

Key Words: social representations, healthcare, sexual and reproductive healthcare.

\section{Resumo}

Esta pesquisa teve como objetivo estudar: descrever o núcleo e periférica de cada uma das representações sociais de saúde sexual e reprodutiva em estudantes da Escola de Enfermagem de Culiacan, núcleo do México, e analisar as diferenças entre homens e mulheres. A abordagem teórica baseia-se nos conceitos da teoria das representações sociais de Moscovici (2000), e os principais conceitos de Waldow cuidados (2004), a saúde sexual 
e reprodutiva (SSA / México, 2006) irá orientar a abordagem teórica deste investigação. Com semi quantitativa estruturada abordagem metodológica aos questionários abordagem estrutural que foram aplicadas. Para a análise da análise da informação foi usada núcleo Abric (1994). Os resultados revelaram três categorias: I) Cuidados gravidez indesejada saúde sexual e reprodutiva no feminino e masculino. II) os cuidados de saúde sexual e reprodutiva na contracepção feminina e masculina. E III) cuidados de saúde sexual e reprodutiva em doenças de transmissão sexual feminino e masculino. Com quatro subcategorias: 1) Relação com vários parceiros, 2) educação sexual ausente, 3) Pensamentos para mim não vai acontecer 4) Álcool e drogas.

Palavras-chave: representações sociais, cuidados, saúde sexual e reprodutiva.

Fecha recepción: Enero 2016

Fecha aceptación: Julio 2016

\section{Introducción}

La salud sexual y reproductiva es el estado de completo bienestar físico, mental y social, y no meramente la ausencia de enfermedad en todas las cuestiones relativas al aparato reproductor y sus funciones y procesos (Naciones Unidas, 1994). Además, es un componente esencial en la capacidad de los adolescentes para transformarse en personas equilibradas, responsables y productivas dentro de la sociedad (MEXFAM, 1995). Los adolescentes tienen necesidades de salud particulares que difieren en aspectos importantes de los adultos. La equidad de género es un componente imprescindible para satisfacer sus necesidades y asegurar la salud sexual y reproductiva a lo largo de todo el ciclo vital (SSA, 2002).

En relación con el conocimiento que los adolescentes sinaloenses tienen de los métodos anticonceptivos, $90.5 \%$ de la población de 12 a 19 años de edad informó haber escuchado hablar de algún método anticonceptivo. Asimismo, la respuesta a esta pregunta por grupos de edad muestra que $85.5 \%$ de los adolescentes de menor edad (12 a 15 años) dijo haber escuchado de algún método, al igual que $94.7 \%$ de los de 16 a 19 años. En contraste, entre 
los adolescentes de 12 a 19 años de edad, $73.9 \%$ en 2000 y $79.4 \%$ en 2006, declararon conocer o haber escuchado hablar de algún método anticonceptivo (ENSANUT, 2012).

Los comportamientos en el cuidado de la salud están enraizados por los conocimientos acumulados y por las representaciones sociales, que tienen un carácter dinámico y que se van construyendo en las diferentes etapas de la vida. A su vez, el mundo simbólico comprende estructuras de pensamiento y marcos de referencia desde los cuales se interpretan los fenómenos que afectan a los sujetos configurando un sistema de pensamiento lógico que es aplicado en las prácticas cotidianas. Cuando el pensamiento lógico está guiado por el mundo simbólico, los fenómenos de la salud y la enfermedad se codifican desde ese marco de referencia, dando lugar a comportamientos específicos que están de acuerdo con su trasfondo explicativo y que resultan muy resistentes al cambio, debido al proceso de objetivación, naturalización y anclaje (Candreva, 2004). Los objetivos que nos hemos planteado en esta investigación son: 1. Describir el núcleo central y núcleo periférico de cada una de las representaciones sociales de la salud sexual y reproductiva en estudiantes de la Escuela Superior de Enfermería Culiacán, México. Y 2. Analizar las diferencias de género entre hombres y mujeres respecto a las representaciones sociales de la salud sexual y reproductiva en estudiantes de la Escuela Superior de Enfermería Culiacán, México.

\section{Desarrollo de contenidos sobre la temática seleccionada}

Por su localización, dentro del territorio nacional el Estado de Sinaloa colinda al norte con los Estados de Sonora y Chihuahua, al sur con Nayarit, al este con Durango y al oeste con el Océano Pacífico y el Mar de Cortés. Su extensión territorial comprende $58092 \mathrm{Km}^{2}$, lo que representa $2.97 \%$ del total nacional, ocupando el decimoséptimo lugar en extensión. Además tiene una superficie insular de $60 \mathrm{Km}^{2}$ y una plataforma continental de 17751 $\mathrm{Km}^{2}$. El Estado de Sinaloa se limita por las coordenadas $22^{\circ} 31^{\prime} 00^{\prime \prime}$, y $26^{\circ} 56^{\prime} 00^{\prime \prime}$ de latitud norte y los $105^{\circ} 24^{\prime} 00^{\prime \prime}$ y $109^{\circ} 27^{\prime} 00^{\prime \prime}$, de longitud oeste del Meridiano de Greenwich (INE, 2015).

El inicio de vida sexual es un episodio crucial en la vida de los individuos porque tiene implicaciones para su futuro, como asumir nuevos roles y patrones de comportamiento que 
tendrán efectos en su salud sexual y reproductiva y que modificarán su desarrollo durante la edad adulta (Stern et al., 2003). El inicio de vida sexual en los adolescentes de 12 a 19 años, incluyendo a aquellos que iniciaron vida sexual pero no recordaron la edad, alcanza un porcentaje de $23.8 \%$, y los porcentajes son diferentes por sexo, $30.6 \%$ entre hombres y $16.8 \%$ entre mujeres. De acuerdo con estos resultados, el porcentaje de adolescentes en Sinaloa que ha iniciado vida sexual es ligeramente mayor que el nacional (23.0\%). Comparando estos datos con los de encuestas anteriores, en donde se excluye a aquellos adolescentes que no recordaron la edad de inicio, en el año $200016.0 \%$ de los adolescentes entre 12 y 19 años de edad declararon haber iniciado vida sexual y en 2006 fue $15.7 \%$ (ENSANUT, 2012).

Del total de adolescentes de 12 a 19 años edad que han iniciado vida sexual, $33.1 \%$ no utilizó ningún método anticonceptivo en la primera relación sexual, porcentaje mayor al nacional (22.9\%). De los que sí usaron algún método, $64.3 \%$ utilizó condón masculino, cifra menor al porcentaje nacional (72.2 \%). Comparando esta información con la de 2006, se observa que $55.2 \%$ de los adolescentes sinaloenses no utilizó ningún método anticonceptivo en la primera relación sexual, mientras que $35.3 \%$ usó condón masculino (ENSANUT, 2012).

En la última relación sexual, el uso de métodos anticonceptivos muestra que $26.3 \%$ de los adolescentes no utilizó ningún método; mientras tanto, de los que reportaron haber usado alguno, $60.3 \%$ utilizó el condón masculino, dato menor al nacional (66.0 \%). La tendencia de utilización de condón masculino en Sinaloa entre la primera y última relación sexual es similar, como la de los que no utilizaron ningún método. Al comparar esta información con la de 2006, 36.2 \% de los adolescentes sinaloenses reportó haber utilizado condón en la última relación sexual (ENSANUT, 2012).

Por lo que se refiere al acceso a condones, $28.4 \%$ de los adolescentes de 12 a 19 años reportó haberlo recibido de forma gratuita en los últimos doce meses, cifra menor a la nacional (32.7\%). El promedio de condones masculinos recibidos fue de 9.5. En lo referente al embarazo de adolescentes en Sinaloa, los resultados muestran que $55.7 \%$ de las 
mujeres de 12 a 19 años con inicio de vida sexual alguna vez han estado embarazadas (ENSANUT, 2012).

En lo que respecta a política social en el estado de Sinaloa se aprobó por el H. Congreso del estado el día 14 de octubre de 2015, la ley de niñas, niños y adolescentes del estado de Sinaloa, donde se establece en el capítulo décimo el derecho a la protección a la salud y la seguridad social. Artículo 45. Niñas, niños y adolescentes tienen derecho a disfrutar del más alto nivel posible de salud, así como a recibir la prestación de servicios de atención médica gratuita y de calidad, de conformidad con la legislación aplicable las autoridades estatales y municipales en el ámbito de sus respectivas competencias en relación con este derecho se coordinarán a fin de (Ley de los derechos de niñas, niños y adolescentes del Estado de Sinaloa, 2015).

Esta ley establece en su fracción VI las medidas tendientes a prevenir embarazos de las niñas y las adolescentes, además de aquellas enfocadas al acceso a oportunidades, la permanencia escolar y la creación de un programa de vida; VII Asegurar la prestación de servicios de atención médica respetuosa, efectiva e integral durante el embarazo, parto y puerperio, así como para sus hijas e hijos, y promover la lactancia materna exclusiva dentro de los primeros seis meses y complementaria hasta los dos años, así como garantizar la información y el acceso a métodos anticonceptivos conforme a su edad, desarrollo evolutivo, cognoscitivo y madurez, que los proteja de posibles riesgos, y en caso de requerirse, con la participación de quienes detenten la patria potestad, tutela o guarda y custodia, para el desarrollo de una maternidad y paternidad responsable, de acuerdo al interés superior de la niñez; XI. Proporcionar asesoría y orientación sobre salud sexual y reproductiva (Ley de los derechos de niñas, niños y adolescentes del Estado de Sinaloa, 2015).

La teoría de las representaciones sociales de Moscovici (2000), y los conceptos principales sobre cuidado de Waldow (2006), salud sexual y reproductiva (SSA/México 2006), guiarán el abordaje teórico de la presente investigación. 
La salud sexual forma parte de la salud del ser humano y se refiere al estado de bienestar de hombres y mujeres para tener una vida sexual placentera y segura. Está encaminada al desarrollo de la vida y de las relaciones personales y no meramente al asesoramiento y la atención en materia de reproducción y de enfermedades de transmisión sexual: Conferencia Internacional de Población y Desarrollo de El Cairo 1994. Se entiende por salud sexual a la capacidad de disfrutar de una vida sexual satisfactoria y sin riesgos que no incluye como elemento indispensable la procreación; en esencia, la sexualidad es una oportunidad para desarrollar los valores de amor, comunicación, responsabilidad y equidad de género: La nueva cultura de la salud sexual (SSR, SSA, 2001).

La salud reproductiva supone un estado de completo bienestar físico, mental y social en lo que respecta a todo lo relacionado con el sistema reproductivo, sus funciones, procesos, y no simplemente a la ausencia de enfermedad o debilidad. La salud reproductiva implica la capacidad de disfrutar de una vida sexual satisfactoria, sin riesgos de procrear, la libertad para decidir hacerlo o no hacerlo, cuándo y con qué frecuencia. El hombre y la mujer tienen derecho a obtener información y acceso a métodos seguros, eficaces, asequibles y aceptables para la regulación de la fecundidad, así como el derecho a recibir servicios adecuados de atención a la salud que permitan el embarazo y el parto sin riesgo y con las mayores posibilidades de tener un hijo saludable (Organización Mundial de la Salud; SSR, SSA, 2001).

Hablar de salud sexual es importante ya que este tema a la vez que aborda los aspectos relacionados con la sexualidad, tiene una orientación básicamente preventiva, de fomento a la salud y de evitar riesgos que favorezcan los embarazos no planeados, las infecciones de transmisión sexual (ITS), VIH/SIDA, y los abortos. En el caso de los adolescentes, los expertos opinan que es mejor utilizar el concepto de salud sexual en lugar de salud reproductiva, ya que no es adecuado favorecer el vínculo sexualidad-reproducción, además de que se sabe que las relaciones sexuales, en la mayoría de los casos, no tienen como objetivo central la reproducción. La salud sexual es uno de los temas más recientes que los organismos que trabajan en el campo de la salud y de la educación en nuestro país están incorporando a sus agendas como resultado de diferentes conferencias internacionales, en 
especial la Conferencia Internacional de Población y Desarrollo de las Naciones Unidas, (ONU, El Cairo, 1999).

Las representaciones sociales como forma de conocimiento aluden a un proceso y a un contenido. En tanto proceso, las RS refieren a una forma particular de adquirir y comunicar conocimientos. Como contenido, a una forma particular de conocimiento que constituye un universo de creencias en el que se distinguen tres dimensiones: la actitud, la información y el campo de representación (Moscovici, 1979).

El esquema triádico de Moscovici da supremacía a la relación de sujeto - grupo (otros sujetos), porque: a) Los otros y las otras son mediadores y mediadoras del proceso de construcción del conocimiento y b) La relación de los y las otras con el objeto físico, social, imaginario o real es lo que posibilita la construcción de significados. Esta concepción, a su vez, ilustra sobre la posición epistemológica en la que se inscribe quien estudia las representaciones sociales. En primer término, se parte de que el conocimiento no es sólo comprensible desde la tradicional concepción que señala la existencia de un conocimiento científico y de un conocimiento cotidiano o del sentido común. En esta concepción se comprende el conocimiento como fenómeno o fenómenos complejos que se generan en circunstancias y dinámicas de diversa índole y cuya construcción está multideterminada por relaciones sociales y culturales (Banchs, 1994).

Este tipo de educación consiste en una estructura particular de orientación de la conducta de las personas, cuya función es dinamizar y regular su acción. Es la orientación global positiva o negativa, favorable o desfavorable de una representación. Su identificación en el discurso no ofrece dificultades ya que las categorías lingüísticas contienen un valor, un significado que por consenso social se reconoce como positivo o negativo, por tanto es la más evidente de las tres dimensiones. La actitud expresa el aspecto más afectivo de la representación, por ser la reacción emocional acerca del objeto o del hecho. Es el elemento más primitivo y resistente de las representaciones y se halla siempre presente aunque los otros elementos no estén. Es decir, una persona o un grupo puede tener una reacción emocional sin necesidad de tener mayor información sobre un hecho en particular (Araña, 2002). 
Waldow define los actos de humanizar, respetar, amar y ser solidario, como una forma de vivir, de ser, de expresarse con una postura ética y estética frente al mundo; de contribuir con el bienestar general preservando la naturaleza, la dignidad humana, la parte espiritual y construir la historia del conocimiento y la vida. Es un proceso interactivo, entre cuidadora y ser cuidado, en el que la cuidadora toma un papel activo para desempeñar acciones de cuidar, mientras que el ser cuidado toma conciencia de su situación, contribuye al cuidado y desempeña un papel menos pasivo al ser responsable de su propio cuidado en situaciones de educación para su salud (Waldow, 2006).

Dicha definición otorga al cuidado una dimensión amplia e integradora, contempla las personas en todas sus dimensiones: biológica, psicológica, social y espiritualmente, así como diversos factores del proceso de la salud y enfermedad en su unicidad, diversidad e interacción constante con el entorno. Además, indica que en los cuidados están las actividades, actitudes y sentimientos considerados como complementarios (Waldow, 2006).

Waldow define el proceso de cuidar como el desarrollo de acciones, actitudes y comportamientos basados en conocimiento científico, experiencia, intuición y pensamiento crítico, realizados para y con el ser cuidado, en el sentido de promover, mantener y/o recuperar su dignidad y totalidad humana. Esa dignidad y totalidad engloba sentido de integridad y la plenitud física, social, emocional, espiritual e intelectual en las fases de vivir y morir; constituye por último un proceso de transformación de ambos: cuidadora y paciente cuidado. Al considerarse al cuidado como un proceso interactivo, existen tres dimensiones: personal, social y profesional (Waldow, 2006).

\section{Material y método}

El presente trabajo de investigación es un estudio cuantitativo no experimental realizado desde un diseño transeccional descriptivo, cuyo objetivo es indagar sobre la incidencia y los valores en que se manifiestan una o más variables. El procedimiento consiste en medir en un grupo de personas u objetos una o generalmente más variables y proporcionar su descripción. Son, por lo tanto, estudios puramente descriptivos que cuando establecen hipótesis también son descriptivos (Hernández, 2014). 


\section{Análisis de la información}

La información recolectada por medio de las técnicas características del enfoque estructural se apoya para su análisis en técnicas cuantitativas (Flament, 1986), y en particular descansa en un análisis multidimensional de tipo factorial. Debido a que este tipo de análisis debe seguir un determinado procedimiento según la herramienta estadística seleccionada, la mayoría de las veces el investigador no se enfrenta al volumen de "datos" que se produce al utilizar métodos y técnicas cualitativas. En efecto, por las técnicas que son utilizadas en la investigación cualitativa (la entrevista, la observación, las preguntas abiertas, los diarios, etcétera), el tipo de dato recogido suele expresarse en cadenas verbales y no mediante valores numéricos (Abric, 1994).

\section{Resultados}

\section{CATEGORÍA I: CUIDADO DE LA SALUD SEXUAL Y REPRODUCTIVA EN EL EMBARAZO FEMENINO Y MASCULINO}

Con cinco sub-categorías: 1) Uso de métodos anticonceptivos, 2) Comunicación con sus padres, 3) Educación sexual, 4) La mujer no se cuida en la relación sexual, 5) El hombre tiene relaciones sexuales con varias parejas. 
Grafo categoría 1: cuidado de la salud sexual y reproductiva en el embarazo femenino y masculino
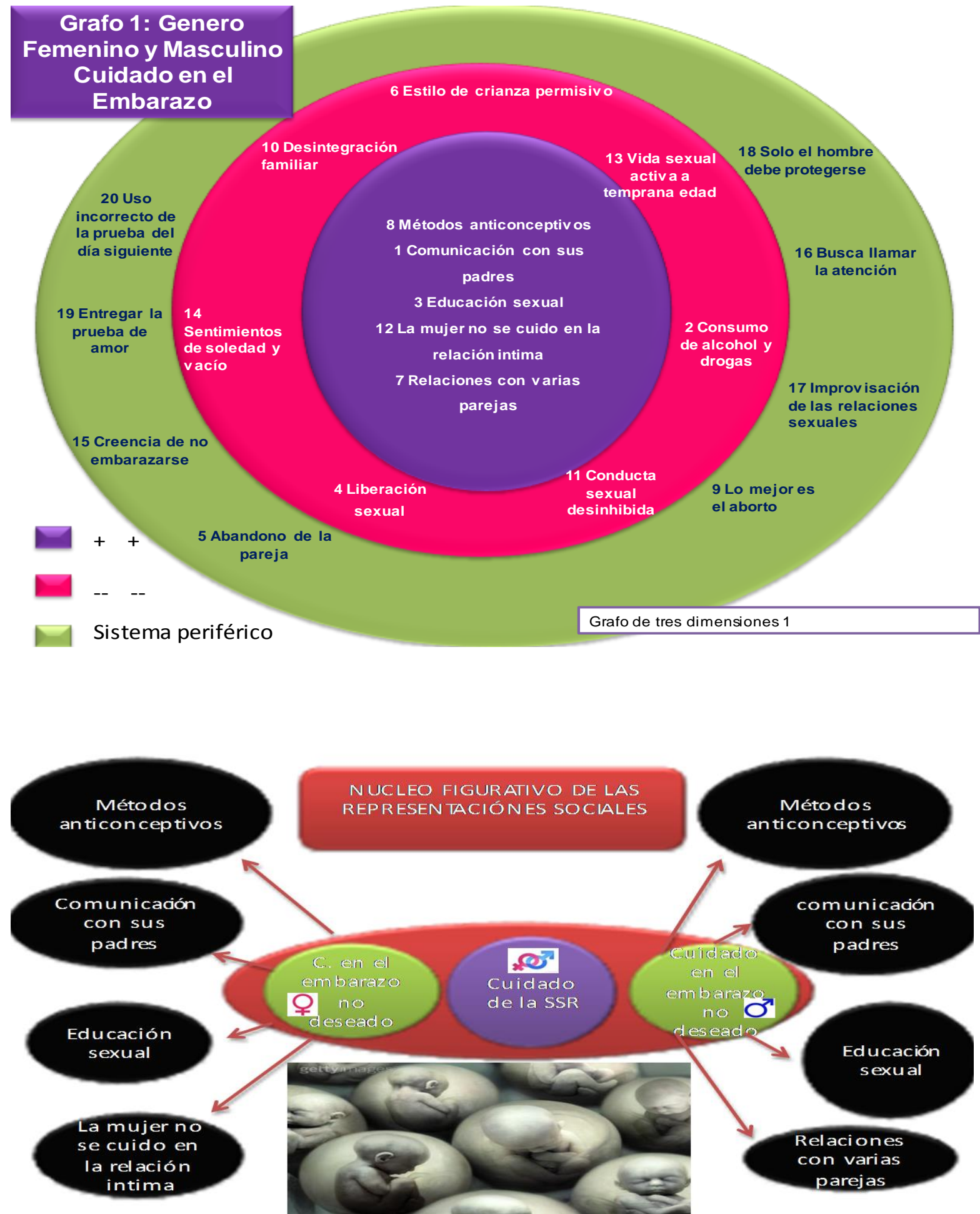

Fuente: grafo 1 
Los resultados del enfoque estructural de las representaciones sociales sobre el cuidado de la salud sexual y reproductiva mostraron que la mayoría de los estudiantes de ambos sexos realizaron asociaciones vinculadas a la categoría de cuidado de la salud sexual y reproductiva en el embarazo femenino y masculino. Se encontró que el núcleo central de dicha representación se forma en torno al cuidado con métodos anticonceptivos, acompañado de ejes periféricos de la representación protectores de la salud, tales como la comunicación con sus padres y la importancia de la educación sexual. También se encontraron diferencias de género; en el caso de las mujeres hubo un nivel significativo representacional pero también periférico en el que la mujer no se cuidó en la relación íntima. Y en el caso de los hombres estuvo presente el tener relaciones con varias parejas. También hay que tener en cuenta la presencia de otros ejes periféricos que sin ser los de mayor importancia, en su conjunto muestran particularidades de la representación: desintegración familiar, inicio de vida sexual activa a temprana edad, consumo de alcohol y drogas, conducta sexual desinhibida, liberación sexual y sentimientos de soledad y vacío.

\section{CATEGORÍA II: CUIDADO DE LA SALUD SEXUAL Y REPRODUCTIVA EN LOS MÉTODOS ANTICONCEPTIVOS FEMENINO Y MASCULINO}

Con cinco sub-categorías: 1) Condón masculino, 2) Es responsabilidad del hombre y la mujer protegerse, 3) Pastillas/DIU, 4) Píldora de emergencia, 5) Coito interrumpido/Condón. 
Grafo categoría 2: cuidado de la salud sexual y reproductiva en los métodos anticonceptivos femenino y masculino
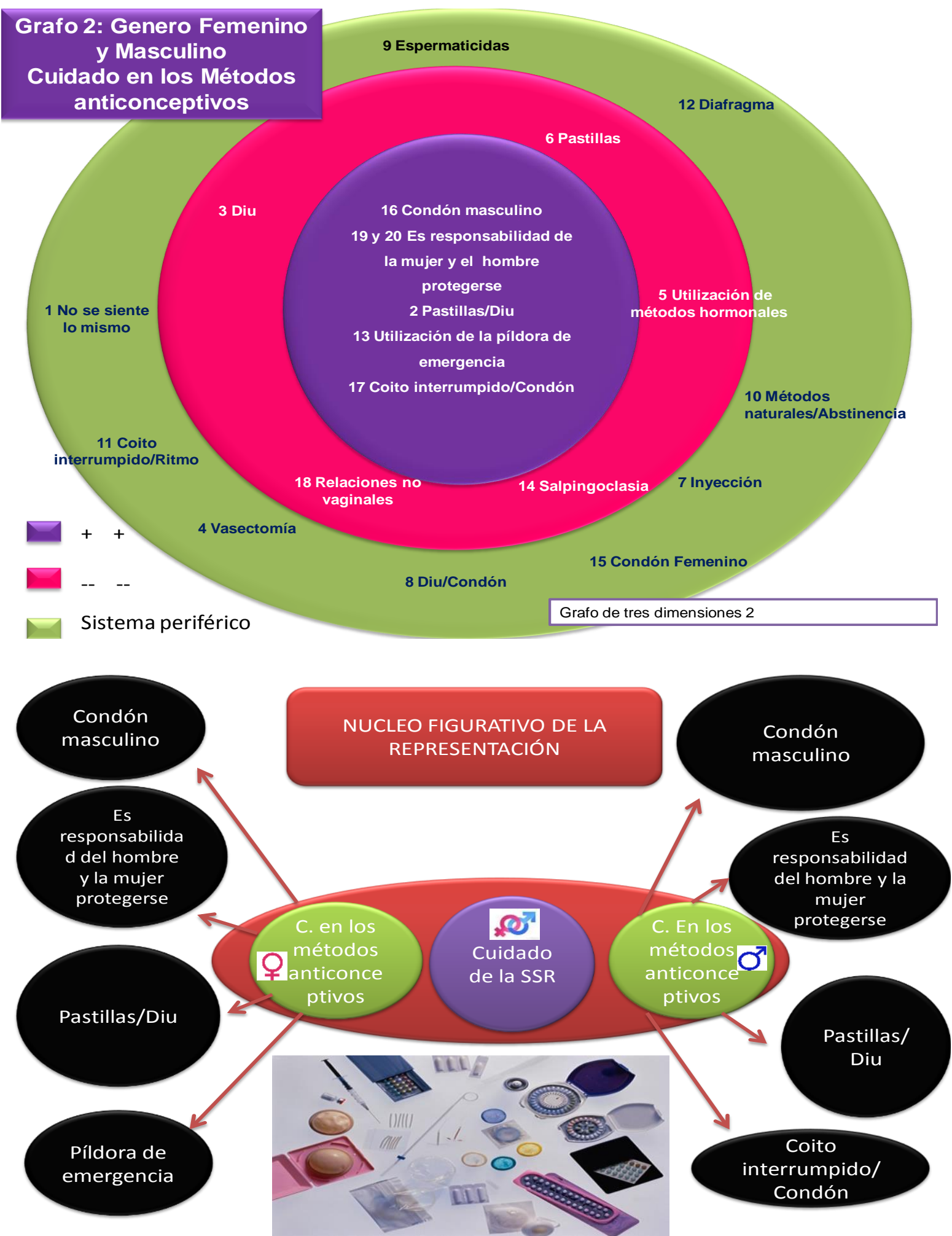

Fuente: grafo 2 
Los resultados del enfoque estructural de las representaciones sociales del cuidado de la salud sexual y reproductiva mostraron que la mayoría de los estudiantes de ambos sexos realizaron asociaciones vinculadas a la categoría de cuidado de la salud sexual y reproductiva en los métodos anticonceptivos femenino y masculino. Se encontró que el núcleo central de dicha representación se forma en torno al cuidado con condón masculino acompañado de ejes periféricos de la representación protectores de la salud, tales como el consenso de que es responsabilidad del hombre y la mujer protegerse por igual y combinar métodos como las pastillas/DIU. También se encontraron diferencias de género; en el caso de las mujeres hubo un nivel significativo representacional pero también periférico en la práctica del uso de la píldora del día siguiente. Y en el caso de los hombres estuvo presente el combinar la práctica del coito interrumpido con el condón. También hay que tener en cuenta la presencia de otros ejes periféricos, que sin ser los de mayor importancia en su conjunto muestran particularidades de la representación: DIU, pastillas, hormonas, salpingoclasia y relaciones no vaginales.

\section{CATEGORÍA III: CUIDADO DE LA SALUD SEXUAL Y REPRODUCTIVA FEMENINA Y MASCULINA EN LAS ENFERMEDADES DE TRANSMISIÓN \\ SEXUAL}

Con cuatro sub-categorías: 1) Relaciones con varias parejas, 2) Educación sexual ausente, 3) Pensamientos de a mí no me va a pasar, 4) Alcohol y drogas. 
Grafo categoría 3: cuidado de la salud sexual y reproductiva femenina y masculina en la prevención de las enfermedades de transmisión sexual
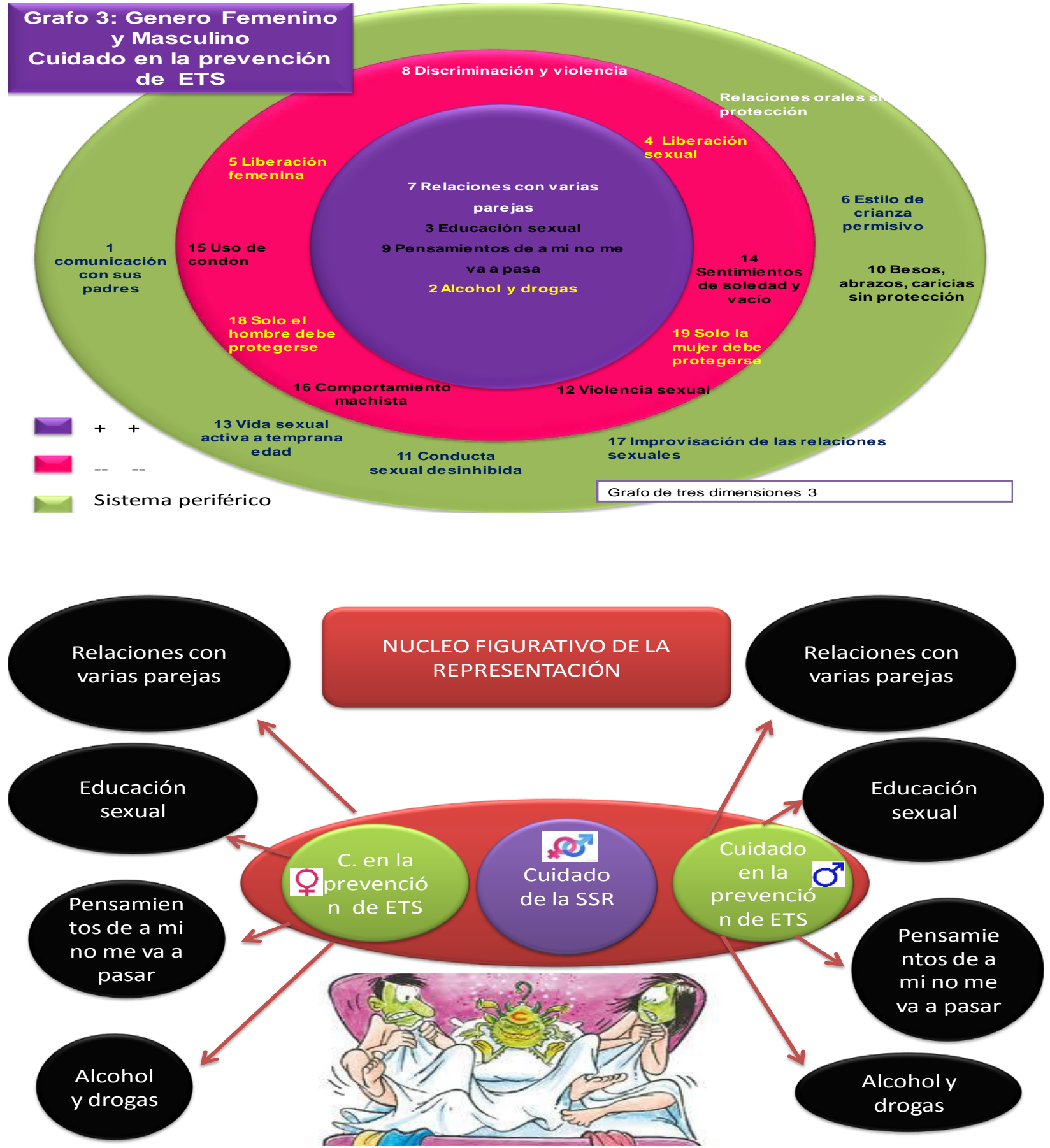

Fuente: grafo 3 
Los resultados del enfoque estructural de las representaciones sociales del cuidado de la salud sexual y reproductiva mostraron que la mayoría de los estudiantes de ambos sexos realizaron asociaciones vinculadas a la categoría de cuidado de la salud sexual y reproductiva en la prevención de las enfermedades de trasmisión sexual. Se encontró asimismo que el núcleo central de dicha representación se forma en torno a las relaciones con varias parejas como principal factor de riesgo, acompañado de ejes periféricos de la representación, tales como la ausencia de educación sexual, pensamientos como a mí no me va a pasar y otros factores de riesgo como el consumo de alcohol y drogas. También hay que tener en cuenta la presencia de otros ejes periféricos que sin ser los de mayor importancia en su conjunto muestran particularidades de la representación: liberación sexual, sentimientos de soledad, discriminación y violencia.

\section{DISCUSIÓN}

La información que reciben los estudiantes de su familia para el cuidado de su salud sexual y reproductiva es muy limitada, es decir, casi no se toca el tema de la sexualidad en el seno familiar (en particular con las mujeres) y en el sistema educativo sigue siendo muy conservadora. Así que la información con la que se cuenta proviene principalmente de internet y otros medios masivos de comunicación.

Collazos (2012), en su investigación Representaciones sociales de la salud sexual de adolescentes sordos y oyentes en la ciudad de Bogotá, Colombia, por parte de la Pontificia Universidad Javeriana, concluyó que los adolescentes representan su salud sexual y sexualidad básicamente como un cúmulo de conocimientos poco flexibles, que se transmiten en espacios formales de educación (colegio, universidad y hogar). En esa transmisión de conocimientos se presentan confusiones y desinformación que tratan de resolver con el apoyo de personas mayores.

De lo anterior se deriva la importancia de conocer, desentrañar y cuestionar el núcleo figurativo de una RS, alrededor del cual se articulan creencias, ya que constituye un paso significativo para la modificación de una representación y, por ende, de una práctica social (Banchs, 1991). 
El cuidado de la salud sexual y reproductiva en estudiantes de ambos sexos se debe principalmente a la utilización del condón masculino como método preventivo por excelencia (Embarazo no deseado y ETS), debido a que no cuentan con la información necesaria para optar por algún otro método.

Las representaciones sociales de los universitarios sobre la abstinencia sexual y los condones como mecanismos de prevención (María R. Estupiñán et al., 2012, Universidad Nacional de Colombia) se vinculan con sus prácticas e interacciones sociales, que después de ser analizadas confluyen en categorías relacionadas con sus características, funciones, uso, valores y creencias. El uso del condón constituye para los universitarios un método efectivo y de fácil acceso que protege y previene el embarazo y las enfermedades de transmisión sexual.

Existen diversas realidades porque la realidad misma proviene de la actividad desarrollada por los individuos, en un proceso que los lleva a formarse su propia visión de la realidad (Ibáñez, 2004).

El núcleo figurativo de la representación social del cuidado de la salud sexual y reproductiva con respecto a las ETS, nos muestra que falta mayor difusión formal (sistema educativo) de la educación sexual. La prevención tiene sentido en la medida en que las mujeres reconozcan la posibilidad de que sus parejas también tienen actividad sexual con otras parejas. Los hombres, por su parte, reconocen tener derecho al placer por el placer y las mujeres reconocen la importancia del placer dentro de una relación afectiva.

Para Fátima Flores (El sida y los jóvenes: Un estudio de representaciones sociales de Fátima Flores Palacios y Martha de Alba, Instituto Nacional de Psiquiatría, 2016), existe cierto grado de información y conciencia en torno al SIDA, que influye en la conducta del grupo estudiado. Esto se traduce en sus actitudes e implica cierto sistema de comportamiento, pero no impulsa intervenciones educativas para difundir los derechos humanos fundamentales relativos al libre ejercicio de la sexualidad sin riesgos, el placer físico y emocional, la libre orientación sexual, la libre elección del número de hijos y la prevención del embarazo. Es una representación social hegemónica. 
La producción del conocimiento de sentido común, en sus conexiones con el conocimiento científico, determinará el núcleo central de la representación social (Abric, 2006).

\section{Conclusiones}

El presente estudio busca difundir información que eduque sobre derechos humanos fundamentales relativos al libre ejercicio de la sexualidad sin riesgos y que conlleva placer físico y emocional, libre orientación sexual, libre elección del número de hijos, prevención del embarazo no deseado y prevención de enfermedades de trasmisión sexual. Asimismo se trata de promover el cuidado de la salud sexual y reproductiva con enfoque de género, brindando mayor difusión a los programas y políticas específicas para mujeres que eliminen las inequidades existentes.

Además se recomienda promover el cuidado a la salud sexual desde el núcleo familiar para mejorar la planeación de la vida sexual activa. La promoción del cuidado de la salud sexual y reproductiva debe estar presente en el sistema educativo nacional como una competencia trasversal y en los planes educativos de enfermería como una unidad de aprendizaje obligatoria.

La Escuela Superior de Enfermería Culiacán de la Universidad Autónoma de Sinaloa, debe tener en cuenta los hallazgos de la presente investigación, para fortalecer la enseñanza aprendizaje de los estudiantes de enfermería en prácticas clínicas y comunitarias del primer nivel de atención a la salud.

Es de suma importancia establecer políticas públicas para verdaderamente prevenir los embarazos no deseados y las enfermedades de trasmisión sexual, así como establecer redes de investigación nacional e internacional del cuidado de la salud sexual y reproductiva que genere la aplicación del conocimiento. 


\section{Bibliografía}

Abric, J., (1994) Metodología de recolección de las representaciones sociales. México DF, Ediciones Coyoacán.

Abric, J., (2006) Prácticas sociales y representaciones. México DF, Ediciones Coyoacán.

Araya, S., (2002) Las representaciones sociales: ejes teóricos para su discusión Cuaderno de Ciencias Sociales 127, San José, Costa Rica, FLACSO.

Banchs, M. et al., (2007) "Imaginarios, representaciones y memoria social"., de Alba (coords.) México, DF, Ed. Arruda.

Banchs, M. (1994). Desconstruyendo una desconstrucción: Representation. Threads of discussion, Electronic Version, 3, Peer Reviewed Online Journal. 1- 20. www.swp.uni-linz.ac.at/content/psr/psrindex.htm

Bachs, M., (2000) Aproximaciones procesuales y estructurales al estudio de las Representaciones Sociales. México DF, Textus sur les représentation sociales, 9, 15

Collazos, (2012). Representaciones sociales de la salud sexual de adolescentes sordos y oyentes en la ciudad de Bogotá. Pensamiento Psicológico, vol. 10, núm. 2, 2012, pp. 35-47. Cali, Colombia. Pontificia Universidad Javeriana.

Dommarco, J., et al. (2012). Encuesta Nacional de salud y nutrición. México, DF, Instituto Nacional de Salud Pública, ENSANUT, 1, 200.

Fernández, P., et al. (2014). Dinámica demográfica 1990-2010 y proyecciones de población 2010-2030. 2014, de CONAPO Sitio web: www.conapo.mx.

Fundación Mexicana para la Planeación Familiar (1995). Perspectiva hacia el siglo XXI de la nueva cultura de la salud sexual. México, Mexfam.

Flores, F. et al. (2006). El SIDA y los jóvenes: Un estudio de representaciones sociales. Salud Mental, vol. 29, núm. 3, mayo-junio, 2006, pp. 51-59 Instituto Nacional de Psiquiatría Ramón de la Fuente Muñiz, DF, México.

Flores, F. et al. (2003). Representación social del SIDA en estudiantes de la Ciudad de México. Salud Pública de México, 45, 8.

Hernández, R. et al. (2014). Metodología de la investigación. Lima, Perú. McGraw-Hill.

Ibáñez, T. (1994). La construcción del conocimiento desde una perspectiva socioconstruccionista. Editorial AVEPS, Caracas, Venezuela, serie Conocimiento, realidad e ideología. 
INE (2015). Contexto geográfico de la entidad federativa. México, DF. En Memoria del Proceso Electoral Federal 2005-2006 (5).

Instituto Nacional de Salud Pública. Encuesta Nacional de Salud y Nutrición 2012. Resultados por entidad federativa, Sinaloa. Cuernavaca, México.

Ley de los derechos de niñas, niños y adolescentes del Estado de Sinaloa (2015).

Marriner, A. (2005). Modelos y teorías en enfermería. Madrid, Elsevier.

Martínez, A. (2005). Educación y prevención del SIDA. Anales de psicología, 21, 8.

MEXFAM (1995). Fundación Mexicana para la planeación familiar, México.

Moscovici, S. (1979). El psicoanálisis, su imagen y su público, Buenos Aires, Ed. Huemul.

Moscovici, S., et al. (1998). Psicología Social: influencia y cambio de actitudes; individuos y grupos. Barcelona, Paidós.

Moscovici, S., et al. (2000). Psicología Social II: pensamiento y vida social. Psicología social y problemas sociales. México, Paidós.

Novel, M., et al. (2005). Enfermería psicosocial y salud mental, Barcelona, España. Editorial: Elsevier Masson.

ONU (1995). Cuarta conferencia mundial sobre la mujer, Beijing.

ONU (1994). Conferencia internacional sobre población y desarrollo. El Cairo, Egipto.

OPS (2000). Promoción de la salud sexual, recomendaciones para la acción, Antigua Guatemala, Asociación Mundial de Sexología.

Stern, C., et al. (2003). Masculinidad y salud sexual y reproductiva: un estudio de caso con adolescentes de la Ciudad de México. Salud Pública, México.

SSA (2002). La salud sexual y reproductiva en la adolescencia: un derecho a conquistar.

México, DF: Dirección General de Salud Reproductiva.

SSA (2002). La salud sexual y reproductiva en la adolescencia: un derecho a conquistar, México, DF.

SSA (2002). Introducción a los métodos anticonceptivos: Información general. México, DF.: Dirección General de Salud Reproductiva.

Triguero, V., et al. (2006). De la representación a la práctica sexual. Un estudio exploratorio de representaciones sociales sobre enfermedades sexualmente transmisibles, sexo y uso de preservativo en jóvenes universitarias y sexoservidoras. Sao Paolo, Brasil, Revista Intercontinental de Psicología y Educación, 8, 19. 
Waldow Vera, Regina (2006). Cuidar: expressão humanizadora da enfermagem. Brasil RJ, Petrópolis. 